Cipango
Cipango

19 | 2012

Le Japon et le fait colonial II

\title{
Henri Bernard-Maître, Pierre Humbertclaude, Maurice Prunier, Présences occidentales au Japon. Du "siècle chrétien » à la réouverture du XIXe siècle
}

Patrick Beillevaire

\section{OpenEdition}

\section{Journals}

Édition électronique

URL : https://journals.openedition.org/cipango/1721

DOI : 10.4000/cipango.1721

ISSN : 2260-7706

Éditeur

INALCO

\section{Édition imprimée}

Date de publication : 30 octobre 2012

ISBN : 978-2-85837-204-7

ISSN : $1164-5857$

\section{Référence électronique}

Patrick Beillevaire, « Henri Bernard-Maître, Pierre Humbertclaude, Maurice Prunier, Présences

occidentales au Japon. Du « siècle chrétien » à la réouverture du xIx siècle », Cipango [En ligne], 19 | 2012, mis en ligne le 06 mai 2014, consulté le 30 juin 2021. URL : http://journals.openedition.org/cipango/ 1721 ; DOI : https://doi.org/10.4000/cipango.1721

Ce document a été généré automatiquement le 30 juin 2021

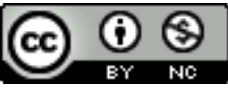

Cipango est mis à disposition selon les termes de la Licence Creative Commons Attribution - Pas d'Utilisation Commerciale 4.0 International. 


\title{
Henri Bernard-Maître, Pierre Humbertclaude, Maurice Prunier, Présences occidentales au Japon. Du " siècle chrétien » à la réouverture du $\mathrm{XIX}$ siècle
}

\author{
Patrick Beillevaire
}

\section{RÉFÉRENCE}

Henri Bernard-Maître, Pierre Humbertclaude, Maurice Prunier, Présences occidentales au Japon. $\mathrm{Du}$ " siècle chrétien » à la réouverture du XIX siècle, édition établie et présentée par Christophe Marquet, Paris, éditions du Cerf, 2011, 432 p. + fig. ISBN : 978-2-204-08525-0.

On ne peut que se réjouir de la parution de ce volume qui rassemble l'essentiel des études sur le Japon du jésuite Henri Bernard-Maître (1889-1975) et du marianiste Pierre Humbertclaude (1899-1984). Publiés entre 1937 et 1942 dans le Bulletin de la Maison franco-japonaise et dans Monumenta nipponica, et pour l'un dans la Revue nationale chinoise, ces articles étaient, il y a lieu de le croire, passablement oubliés et difficiles à trouver (ceux de Monumenta nipponica sont toutefois accessibles en ligne). Le troisième auteur, Maurice Prunier ( ?-1944), n'est présent que pour une brève étude de quatre pages, généreusement illustrée, qui se trouve incluse dans un long article de BernardMaître. Elle traite de peintures faites par des artistes japonais sur le modèle de la peinture italienne de la Renaissance, avec parfois, peut-être, une influence de l'iconographie bouddhique, qui furent utilisées comme « peintures à fouler au pied » ou fumi-e. Outre l'avantage qu'il y a à disposer en un volume de cet ensemble de textes qui se font écho et se complètent sur de nombreux points, ce recueil offre aussi celui de donner à voir en couleur, avec une bonne définition et en format plus grand, les 
illustrations en noir et blanc et de piètre qualité qui accompagnaient les publications originales. Le texte de ces dernières a aussi fait l'objet d'un travail de révision éditoriale qui porte principalement sur les dates, les transcriptions et la traduction des citations. Des compléments d'information sont apportés en note par l'éditeur.

Dans son introduction, Christophe Marquet s'attache à présenter le parcours scientifique et l'œuvre de Bernard-Maître et d'Humbertclaude. Il rappelle notamment comment le premier, qui fut d'abord sinologue, et dont la bibliographie est fort abondante, en est venu à s'intéresser étroitement au Japon au cours de ses recherches sur la littérature chrétienne en Chine et, en particulier, sur l'œuvre de Matteo Ricci. Il donne également une idée des recherches japonaises contemporaines qui servirent d'appui aux deux auteurs, au premier rang desquelles celles de Anesaki Masaharu et de Okamoto Yoshitomo. Bernard-Maître collabora du reste avec ce dernier pour la publication d'une édition critique du manuscrit de Luis Fróis sur l'ambassade japonaise qui s'est rendue à Rome en 1582 .

3 Les articles sont regroupés sous trois intitulés : "Le siècle chrétien ", "Les éditions jésuites au Japon» et "La présence française au Japon avant la réouverture du $\mathrm{XIX}^{\mathrm{e}}$ siècle ». Le premier, qui couvre la moitié du livre, réunit trois articles de BernardMaître : « Les débuts des relations diplomatiques entre le Japon et les Espagnols des îles Philippines ", «Les premiers rapports de la culture européenne avec la civilisation japonaise » et «Infiltrations occidentales au Japon avant la réouverture du XIX siècle ». Sous le deuxième intitulé, on trouve deux articles d'Humbertclaude, «La littérature chrétienne au Japon » et « Le Spiritual shugyō no Manual, ou la presse jésuite au Japon et l'Index », et deux de Bernard-Maître, "Intermédiaires chinois » et "Traductions chinoises d'ouvrages européens au Japon durant la période de fermeture (1614-1853)». La troisième et dernière partie du livre est composée d'un article de Bernard-Maître, «Le Japon et la France à l'époque de la Renaissance (1545-1619) » et de deux articles d'Humbertclaude, «La France au Japon avant 1854 : contribution à l'étude des notions que le Japon avait sur la France et de la pénétration des idées françaises au Japon avant l'ouverture de ce pays » et "Les Français au Japon au XviI e siècle ». Tous ces articles sont d'une grande densité et pourvus d'abondantes références bibliographiques, lorsqu'ils ne portent pas directement sur un matériau bibliographique. La multiplicité même des questions savantes qu'ils abordent nous expose d'évidence à ne pouvoir ni en résumer l'ensemble ni en faire valoir tout l'apport.

Bernard-Maître, l'auteur des trois premiers articles, se préoccupe d'abord de démêler l'écheveau des relations entre les différents acteurs européens présents au Japon, marchands ou missionnaires. Empreints d'une pieuse émulation ou de triviales rivalités étatiques et commerciales, les rapports des Européens avec les Japonais furent presque continûment grevés de tensions ou d'incidents, et bien sûr marqués par l'instabilité politique et la conflictualité de cette période charnière de l'histoire japonaise. Au reste, il n'est pas aisé d'isoler le zèle des missionnaires de l'appétit commercial de leurs coreligionnaires, voire de leurs projets militaires. Des attentes des uns et des autres, de leurs dissensions aussi, les seigneurs japonais - à commencer par les plus puissants, d'Oda Nobunaga aux Tokugawa - surent jouer au gré de leurs intérêts et de leurs ambitions. Avec toujours le souci du contexte historique, Bernard-Maître relate les débuts de la présence portugaise et de l'implantation jésuite, cet «âge d'or du christianisme » que vint soudainement assombrir, le 24 juillet 1587, l'ordre que Toyotomi Hideyoshi donna aux prêtres de quitter le pays dans un délai de vingt jours. 
Ordre suivi d'assez peu d'effets, mais qui présageait de rapports de plus en plus rudes avec les dirigeants du pays, jusqu'à l'interdiction du christianisme et à l'exclusion des Européens. Seuls les Hollandais, on le sait, continuèrent à bénéficier, sous de rigoureuses contraintes, d'un droit de commerce avec le Japon. Les sous-chapitres sur le port cosmopolite de Sakai, havre de paix accueillant aux Européens, et sur Kyōto, capitale en vacance d'autorité politique où François Xavier échoua à se faire accepter, éclairent opportunément l'environnement social et intellectuel qui fut celui des tout premiers missionnaires et marchands. Le déclin rapide de Sakai, au profit d'ōsaka, et l'hostilité patente des moines bouddhistes à Kyōto furent l'une des causes du repli des Jésuites dans l'île de Kyūshū. Ils se réinstalleront néanmoins à Kyōto dès 1569. BernardMaître ne s'attarde guère sur les relations des Jésuites avec les «daimyō chrétiens ", sujet sur lequel il renvoie notamment à l'étude de Michael Steichen parue en 1904. Le développement du commerce japonais avec l'Asie du Sud-Est, concomitant de la présence des Européens au Japon, est un phénomène qui a eu certainement plus de conséquences, sur le long terme, qu'il n'y paraît. Il est l'objet du très intéressant souschapitre intitulé «Expansion dans les mers du Sud ». Mais l'apport principal de l'auteur à l'historiographie concerne les difficultés rencontrées par les Espagnols pour établir des relations diplomatiques avec le Japon. Ses recherches font ici pendant à celles que l'historien britannique C. R. Boxer, aujourd'hui référence majeure sur le «siècle chrétien » du Japon, menait sur les Portugais dans les mêmes années.

Légitimée par les incertitudes, faute d'une détermination précise des longitudes, dans le partage du monde hâtivement fixé par le traité de Tordesillas (1494), la couronne espagnole ne tarda pas, de son avant-poste philippin, à contester le monopole sur les relations avec le Japon revendiqué par le Portugal. Cette prérogative se trouvait pourtant avalisée, en quelque manière, par l'attribution du Japon au diocèse de Macao décidée en 1575 par le pape Grégoire XIII. En 1580, l'ascension de Philippe II d'Espagne au trône du Portugal, sous le nom de Philippe I, put faire naître l'espoir que se réduirait l'antagonisme entre les deux nations. Mais il n'en fut rien. Le souverain espagnol s'en tint à sa promesse de respecter l'autonomie administrative du Portugal, en particulier dans ses affaires coloniales et son commerce maritime. La situation juridique du Japon demeurant floue au regard des prétentions de l'un et l'autre pays, les Espagnols s'efforcèrent donc d'y prendre pied, par la voie de la Nouvelle Espagne et de Manille, et de s'y faire admettre comme partenaires commerciaux, à l'instar des Portugais qui chaque année envoyaient de Macao une caraque (não), véritable "banque flottante", prisée des Japonais. L'auteur, citant abondamment les sources de l'époque, rend compte de leurs démarches incertaines et des divers aléas survenus avant qu'ils n'atteignent leur objectif. Admis tardivement, alors que Hollandais et Anglais se posaient déjà en rivaux des puissances ibériques, leur position ne sera jamais que précaire. Leur bannissement intervint d'ailleurs dès 1624, bien avant celui des Portugais, en 1639. L'esprit de concurrence n'épargna pas non plus les relations entre missionnaires portugais et espagnols. Non pas tant au sein de la Compagnie de Jésus, à laquelle le Saint-Siège avait confié la mission du Japon, qu'entre ses membres et ceux des ordres mendiants, Franciscains, Dominicains et Augustins. Animés par leur loyauté première envers Rome, les Jésuites essayèrent, pour leur part, de s'affranchir de la mainmise des autorités civiles et de l'attachement à l'appartenance nationale, mais les arrangements qu'ils obtinrent ne furent qu'occasionnels. L'accès au Japon via Goa et Macao, pour lequel il était requis de s'embarquer à Lisbonne, fut ainsi réservé en principe, sinon aux seuls missionnaires portugais, du moins à ceux qui acceptaient l'hégémonie du Portugal 
sur les affaires de l'Extrême-Orient. Pour certains jésuites espagnols, ou ceux des autres pays également touchés par l'exclusivisme portugais, notamment les Italiens, le chemin du Japon passa donc par le Mexique et les Philippines. Ce n'est guère sur eux, cependant, que le gouverneur et les marchands de Manille purent compter pour nouer des relations officielles avec Toyotomi Hideyoshi ou se faire reconnaître comme partenaires commerciaux. Assujettis à la couronne d'Espagne et impatients de s'implanter eux aussi au Japon, les moines des ordres mendiants leur furent de plus précieux auxiliaires. Bernard-Maître, exégète méticuleux, appréhende au plus près des événements le climat de confusion et de défiance qui conduisit ces derniers à s'associer étroitement, bon gré mal gré, aux démarches diplomatiques espagnoles. Le caractère hasardeux de celles-ci, notamment en raison du manque de fiabilité des intermédiaires japonais, à l'instar de l'aventurier Paul Harada Kiemon, est illustré par l'échec, ou semi échec, des missions du dominicain Juan Cobo, en 1592, et du franciscain Pedro Bautista, l'année suivante. À tout le moins, les Espagnols purent alors prendre conscience que les nombreux titres dont se prévalait leur souverain n'impressionnaient guère Toyotomi Hideyoshi, et que celui-ci, en revanche, entendait avoir la pleine tutelle sur le négoce avec les îles Philippines, s'il ne projetait pas de les conquérir. Malgré l'injonction du pape Clément VIII à tout le clergé, suivie de celle du roi d'Espagne Philippe III (Philippe II du Portugal) à ses sujets, demandant que soit respectée l'appartenance de la Chine et du Japon à la zone d'influence du Portugal, et par conséquent que les missionnaires empruntent la route maritime Lisbonne-Goa-Macao, la crainte que leur dessein apostolique ne se trouvât entravé par les autorités portugaises était telle chez les moines espagnols que la plupart préférèrent passer outre et gagner l'Extrême-Orient par les Philippines. Le développement d'un épiscopat japonais a lui aussi pâti de la rivalité entre l'Espagne et le Portugal, comme le montrent les nombreuses négociations et controverses rapportées par Bernard-Maitre, ou son évocation du parcours mouvementé et inabouti de l'archevêque de Myre, mort discrètement à Paris. Sur ce sujet, déjà étudié par le jésuite Joseph de Moidrey, l'auteur apporte beaucoup de compléments et quelques corrections. Avec la création par le pape Grégoire XV, en 1622, de la congrégation de Propaganda Fide, puis l'institution de vicariats apostoliques, le Saint-Siège commencera à s'affranchir, non sans difficulté, du padroado portugais pour lui-même prendre en main l'organisation des activités missionnaires. Pour le Japon, d'où missionnaires et christianisme étaient déjà bannis, il était trop tard. Ce n'est qu'au $\mathrm{XIX}^{\mathrm{e}}$ siècle, avec l'attribution de la mission du Japon à la Société des missions étrangères de Paris, que le Saint-Siège aura à nouveau la possibilité d'y exercer ses prérogatives. L'auteur s'éloigne quelque peu des faits, toutefois, lorsqu'il écrit qu'Augustin Forcade, prêtre de cette société élevé à la dignité d'évêque du Japon en 1846, a pu «effectuer les grands projets de l'archevêque de Myre ", alors qu'il ne lui fut jamais permis de poser un pied dans ce pays.

6 Bernard-Maître ne manque pas de s'interroger sur le contraste toujours surprenant entre le bon accueil initial fait à la civilisation occidentale et la brutale répression qui accompagna, seulement quelques décennies plus tard, l'interdiction du christianisme et le repli défensif du Japon sur son espace insulaire. La détermination mise à éradiquer le christianisme apparaît d'autant plus inattendue, selon lui, qu'il tendait à conforter les vertus confucéennes imprégnant la société japonaise et, plus encore, qu'il « collabora pour une part non négligeable à l'exaltation des traditions nationales ». Ces lignes, il ne faut pas l'oublier, furent écrites à une époque de nationalisme exacerbé et de suspicion à l'encontre des chrétiens. Mais, plutôt que de rechercher dans le décours des 
événements une explication à la radicalité de ce retournement, c'est en regardant du côté de la Chine de cette époque et des débats au sein du confucianisme qu'il dégage ce qui lui semble en avoir été le ressort profond, d'ordre idéologique: "l'influence stérilisatrice » du néo-confucianisme de Zhu Xi sur la pensée des dirigeants japonais. Le fait que les daimyō de Satsuma aient été très tôt acquis à cette doctrine «matérialiste et areligieuse " expliquerait d'ailleurs l'insuccès de François Xavier dans ce fief. Son application à la situation du Japon par Hayashi Razan, conseiller auprès de Tokugawa Ieyasu et de ses trois premiers successeurs, entraîna à la fois un complet rejet de la civilisation occidentale et une "immobilisation de la pensée japonaise ", en même temps qu'elle apporta une assise idéologique au régime shōgunal et à la domination de la classe guerrière. La curiosité pour l'Europe recommencera à se manifester plus librement un siècle plus tard, sous le règne de Tokugawa Yoshimune, avec le relâchement de la censure sur les livres importés, avant de connaître une véritable renaissance au XIX ${ }^{e}$ siècle, par exemple avec le penseur Yoshida Shōin. Si BernardMaitre revient plusieurs fois dans ses articles sur l'influence, pour lui aussi cruciale que néfaste, de la doctrine de Zhu Xi, il consacre un nombre de pages plus important encore à montrer que l'humanisme européen de la Contre-Réforme «ensemencé » au Japon par les Jésuites trouva une résonnance, sinon un relais, dans la doctrine « personnaliste » et « spiritualiste » de Wang Yangming (Ō Yōmei, 1472-1528). En Chine, peu de temps auparavant, Matteo Ricci avait précisément cherché à contrecarrer l'influence de Zhu Xi, d'un côté, en tirant parti des affinités entre la doctrine de Wang Yangming et l'humanisme chrétien, de l'autre, en revenant aux textes canoniques du confucianisme pour montrer la conciliation possible entre ses préceptes et ceux du christianisme. Outre l'estime que lui valurent sa maîtrise de la langue et son érudition, la visée même de ses commentaires n'était pas restée sans effet sur la pensée des lettrés chinois. Conscient de l'importance de ces débats, l'Italien Alessandro Valignano, visiteur général des missions jésuites en Orient, qui séjourne une première fois au Japon de 1579 à 1582, entreprit d'introduire au Japon les écrits de Matteo Ricci. Mais, contrairement aux Jésuites de Chine, ceux du Japon ne trouvèrent pas motif à s'impliquer dans cette réflexion. L'absence d'un corps de lettrés comparable à celui de la Chine explique pour une large part leur attitude. Mais sans doute avaient-ils aussi des raisons de ne pas s'inquiéter, confiants dans les "affinités profondes de l'âme japonaise avec le christianisme", devant le nombre des convertis et la relative bénignité de la critique bouddhique. Bernard-Maitre insiste beaucoup sur le stimulus que fut pour la culture japonaise l'arrivée de nombreux Chinois à la fin du régime des Ming, et avec eux l'introduction au Japon d'un volume considérable de livres. Parmi ces exilés hostiles aux Mandchous se trouvaient des lettrés qui contribuèrent à la découverte des idées de Wang Yangming et, plus souterrainement, à l'acclimatation d'un questionnement philosophique dans lequel transparaissait l'influence de la culture occidentale et des idées chrétiennes. Nakae Tōju le premier, dont la réflexion semble directement empreinte de notions chrétiennes, comme l'a relevé Anesaki Masaharu, puis son disciple Kumazawa Banzan, malgré un antichristianisme peut-être dicté par la prudence, et d'autres après eux, tels Marubashi Chūya ou Yūi Shōsetsu, se firent les propagateurs de ce nouveau courant de pensée sur le sol japonais. L'auteur, assuré de l'existence d'un «humanisme universel » au-delà de la diversité de forme, distingue ainsi trois voies à travers lesquelles a pu se poursuivre au Japon la découverte des "affinités intellectuelles et culturelles » entre l'Occident et l'Extrême-Orient (ou, dans une autre formulation plus engagée, par lesquelles «la culture occidentale a pu 
continuer son influence bienfaisante dans la civilisation japonaise »): les livres chrétiens, ou reflétant une influence chrétienne, introduits par les réfugiés chinois, les écrits des lettrés de l'école Tong-lin dans lesquels avaient été ensemencées les « vérités venues de l'Europe ", et surtout la pensée de Wang Yangming qui gagna bientôt en influence dans les élites de l'époque d'Edo. Dans un autre article, Bernard-Maître examine les ouvrages européens traduits en chinois, traitant de religion ou de science, qui circulèrent au Japon durant cette époque. Outre les données bibliographiques qu'elle fournit, sans doute assez exhaustives, son étude nous renseigne concrètement sur la pratique et l'évolution des critères de la censure, du rejet de toute référence à l'Occident à une attitude plus souple qui laissa place aux informations générales et aux connaissances scientifiques, à commencer par celles relatives à l'astronomie et aux mathématiques. Paradoxalement, c'est au Japon que les intellectuels chinois purent redécouvrir, à partir de la fin du XIXe siècle, beaucoup de ces ouvrages que la censure de leur pays avait plus sûrement fait disparaître.

Plus encore que pour Bernard-Maître, il est difficile de faire justice aux recherches minutieuses de Pierre Humbertclaude. Celles-ci portent, d'une part, sur la littérature imprimée par les Jésuites du Japon, d'autre part, sur ce que les Japonais connaissaient de la France avant les premiers traités. On est évidemment frappé par l'ampleur, la créativité et la qualité du travail d'imprimerie, et aussi de reliure, accompli par les Jésuites et leurs assistants en une vingtaine d'années. Pas moins de vingt-cinq livres virent ainsi le jour (chiffre de Kōda Shigetomo, voir ci-après), sans compter des impressions non brochées. L'importation d'une presse européenne au Japon fut une initiative de Valignano qui l'apporta avec lui lorsqu'il revint au Japon en 1588 (il repartira en 1592, mais séjournera une troisième fois au Japon de 1598 à 1603). Jusquelà avaient circulé, sous forme de copies manuscrites, de multiples traductions japonaises de textes chrétiens, en même temps qu'étaient importés des livres européens, parmi lesquels certains introduisaient aux auteurs de l'Antiquité, socle de l'éducation humaniste que les Jésuites, dans un premier temps surtout, concevaient comme une propédeutique au christianisme. Au moins une grammaire et un dictionnaire, ou différents états de ceux-ci, furent aussi rédigés pour aider les nouveaux missionnaires dans l'apprentissage du japonais. L'essentiel de la littérature imprimée par les Jésuites au Japon est bien sûr composé d'ouvrages, agrégeant souvent des textes hétérogènes, destinés à l'enseignement de la religion chrétienne et à la formation d'un clergé indigène, objectif primordial pour Valignano: ouvrages catéchétiques pour les aspirants, ouvrages doctrinaux pour les convertis ou pour les prêtres, ouvrages d'ascèse, instructions pour l'administration des sacrements, réfutations des critiques portées contre le christianisme, évangiles, apologies de la foi chrétienne, sermons, etc. Certains sont en rōmaji, une quinzaine, d'autres en kana et en kanji, une dizaine. Humbertclaude ne fournit pas de chiffres; il n'aborde pas non plus les aspects techniques de l'impression. Mais sur ces questions, on pourra lire avec beaucoup d'intérêt l'article de Kōda Shigetomo dont il est le traducteur et qui a paru dans Monumenta nipponica deux ans plus tard, en 1939 ( Notes sur la presse jésuite au Japon et plus spécialement sur les livres imprimés en caractères Japonais »). Les imprimeurs jésuites en vinrent rapidement à utiliser conjointement des caractères mobiles métalliques et des caractères en bois pour allier rōmaji et écriture japonaise. Par l'emploi de caractères cursifs, de handakuon, de furigana et d'autres innovations comme l'impression en noir et rouge, ils firent montre d'une avance technique certaine par rapport aux presses japonaises de la région de Kyōto qui utilisaient également, 
depuis peu, des caractères mobiles. L'imprimerie jésuite fut successivement établie à Kazusa, Amakusa et Nagasaki. C'est probablement en 1591 que fut imprimé le premier de ces ouvrages, un recueil d'extraits des Actes des saints (Santosu no go-sagyō) en rōmaji, suivi d'un petit vocabulaire japonais-portugais. Certains ouvrages des Jésuites du Japon furent cependant imprimés avant cette date à Macao ou même à Lisbonne, comme le catéchisme de Valignano, "où se réfutent les sectes japonaises ", de 1586. Anjirō, ou Yajirō, le guide de François Xavier, fut peut-être l'auteur d'un premier catéchisme japonais, aujourd'hui perdu, imprimé à Goa et écrit en rōmaji, ce qui ferait de lui le pionnier de ce mode d'écriture. L'analyse que fait Humbertclaude de cette littérature révèle une complexe intertextualité : contenus composites, emprunts aux ouvrages européens ou japonais, variations éditoriales. Mais de la presse jésuite sortirent aussi des ouvrages dits profanes, principalement des ouvrages de langue, grammaires et dictionnaires. Valignano, qui recommandait l'adaptation à la culture indigène contrairement au Portugais Francisco Cabral, son prédécesseur à la tête de la mission du Japon - faisait de la connaissance du japonais un impératif, et à cette fin avait été créée à Usuki une école de formation. Parmi ces ouvrages, on sait l'importance qu'a prise la grammaire de João Rodriguez, datée 1604 sur la page de titre, mais achevée quatre ans plus tard, pour la connaissance de la langue japonaise de l'époque, en particulier de la langue parlée, en raison de l'abondance des matériaux littéraires, transcrits en rōmaji, qu'elle contient. La littérature profane comprend aussi plusieurs récits, ou "monogataris", écrits par des convertis et présentant une tonalité édifiante qui les destinait à l'éducation des enfants. D'autres ouvrages ont été conçus à l'usage des Européens pour leur permettre de se familiariser avec la culture japonaise : parmi ceux-ci on trouve le Heike monogatari adapté sous forme de dialogues, comme l'affectionnaient les Jésuites, six volumes d'extraits du Taiheiki et le recueil de poésies japonaises et chinoises Wakan rōeishū. Le fameux ouvrage intitulé Fables d'Ésope, dont le contenu est en réalité assez éloigné d'Ésope et de ses fables, s'adressait, lui, à un lectorat japonais. Il fut d'ailleurs le seul à ne pas être ensuite proscrit par la censure. En raison de leur objet, les recherches d'Humbertclaude dans ce premier article offrent moins de pénétration dans la culture japonaise que celles de Bernard-Maître. Il nous semble, cependant, que la littérature produite par les Jésuites du Japon, si on la compare à celle de leurs confrères de Chine, témoigne par sa nature même d'une capacité de réception ou d'une perméabilité aux idées étrangères propres à la culture japonaise. Dans un autre court article, Humbertclaude retrace, documents de l'instruction à l'appui, les circonstances qui ont amené Diego Collado, un dominicain du Japon contrarié par la domination des Jésuites, à dénoncer à l'Index, en 1630, un ouvrage imprimé à Nagasaki en 1607, le Spiritual xuguio (shugyō) no manual. L'ouvrage, écrivait-il, « risquait de causer l'introduction de doctrines mauvaises dans la nouvelle chrétienté ». La Propagande finit par lui donner gain de cause, mais on ne trouva point d'exemplaires à détruire, lui-même s'étant gardé d'envoyer celui qui était en sa possession. Le troisième article d'Humbertclaude, sur la connaissance que le Japon avait de la France avant 1854, est un peu le pendant thématique, mutatis mutandis, de celui de Bernard-Maître sur la connaissance du Japon en France à l'époque de la Renaissance. Son matériau bibliographique est cependant d'une tout autre densité. On ne peut qu'être fasciné par l'étendue et la précision des informations qu'il recèle, de la géographie aux sciences, en passant par l'histoire, les institutions, la littérature, quoique très marginalement, et les beaux-arts. Le Zōyaku sairan igen (1804) de Yamamura Shōei et le Yochi Shiryaku (1826) d'Aoji Rinsō, pour ne mentionner que deux 
livres parmi tant d'autres, contiennent ainsi de très étonnantes précisions quantitatives et descriptives sur Paris et les provinces françaises. On y apprend aussi que le roi guérissait les écrouelles et qu'à sa mort le trône n'était jamais vacant (« Le roi ne meurt jamais »). Du Français, Aoji Rinsō dresse ce portrait flatteur : «Chez lui les lignes sont parfaites; il est sagace, vif et courageux ; ses manières sont courtoises, sa parole facile et claire. Affable et hospitalier, il accueille volontiers l'étranger et fait preuve envers lui de la plus grande cordialité. »

8 Recueil d'articles de recherche, Présences occidentales au Japon n'a pas le caractère organisé et systématique d'une monographie. Certains aspects de cette première époque des relations entre Européens et Japonais n'y sont qu'évoqués. Mais c'est assurément un livre d'une rare richesse, dont nous n'avons pu donner ici qu'un aperçu très fragmentaire. Sa lecture se recommande tant aux étudiants, auxquels elle permettra d'aborder la culture et l'histoire du Japon sous des angles originaux, qu'aux enseignants et chercheurs, qui y trouveront une mine d'informations érudites propres à nourrir leur réflexion.

\section{AUTEUR}

\section{PATRICK BEILLEVAIRE}

CNRS-EHESS, UMR 8173 